\title{
Money vs. Social Life: Why People Choose Not to Use Facebook Messenger Payment
}

\author{
Lingyuan $\mathrm{Li}$ \\ Clemson University \\ lingyu2@g.clemson.edu
}

\author{
Guo Freeman \\ Clemson University \\ guof@clemson.edu
}

\begin{abstract}
As money and mobile $P 2 P$ payment experiences have become an emerging research agenda in $\mathrm{HCI}$, prior studies have focused on the factors that promote the use of $P 2 P$ payment services as well as related design implications. Yet, few have investigated why people decide not to use such services (e.g., technology non-use) and how people perceive the increasing trend of integrating P2P payments with social media services. To explore these issues, we identified factors that hindered people from using Facebook Messenger payment and their perceptions of integrating of $\mathrm{P} 2 \mathrm{P}$ payments with social media based on 158 social media posts and eight interviews. This study not only extends our existing understanding of technology non-use through the lens of Facebook payment but also helps HCI researchers and designers innovate emerging financial technologies and better understand the global phenomenon of all-in-one design.
\end{abstract}

\section{Introduction}

Over the past decade, we have witnessed the increasing popularity of digital peer-to-peer (P2P) payment (i.e., performing one to one online financial transactions via a digital or mobile device [1]). Popular payment apps such as Venmo, Cash App, and Zelle have been widely used for a variety of personal financial services, including splitting bills, sharing rent, and transferring between friends [1, 2, 3]. Especially for millennials, mobile P2P payments have been seamlessly integrated with their daily lives - "Venmo" has even become a verb [4].

In addition to these professional payment apps, several social media giants (e.g., Facebook in the USA and WeChat in China) also endeavor to offer P2P payment feature within their own ecosystems. WeChat has emerged as the predominant $\mathrm{P} 2 \mathrm{P}$ payment app in China. Business Insider reported that at least 900 million people use WeChat Pay on a monthly basis in
2018 [5]. In Q4 2019, Tencent reported that WeChat Pay had 800 million monthly active users, and one billion WeChat Payment commercial transactions took place per day [6]. Unlike WeChat Pay, Messenger payment has not been successful in the P2P payment market. In 2015, Facebook built a P2P payment feature into its instant message system - Messenger (Fig 1 and 2). Without downloading a standalone app or interrupting the chat process, Facebook users are able to send and/or receive money from a friend within Messenger; even for people who do not have a Facebook account, they still can access and use Messenger payment by using their phone number to sign up [7,8]. Facebook also had more than 1.4 billion users in 2015, when Messenger payment rolled out [9], and the number of monthly active users in Messenger surpassed 600 million [10]. This means that Messenger payment already faced a tremendous user base without struggling to attract new users from scratch. However, despite its seeming convenience and simplicity, in 2017, Statista showed that $79 \%$ of respondents did not use this service [11]. In June 2019, Facebook had to shut down Messenger payment in UK and France [12].

As both WeChat and Facebook are social media platforms that incorporate a built-in payment feature, why one is successful and well received by users while the other fails are unclear. Therefore, we believe that an investigation of the reasons and challenges behind the fall of Messenger payment worthy of research attention in $\mathrm{HCI}$. As money and mobile $\mathrm{P} 2 \mathrm{P}$ payment experiences have become an emerging research agenda in HCI [13], prior studies have focused on the factors that promote the use of $\mathrm{P} 2 \mathrm{P}$ payment services as well as related design implications $[14,15,16]$. Nevertheless, few have investigated why people decide not to use such services (e.g., technology non-use) and how people perceive the increasing trend of integrating $\mathrm{P} 2 \mathrm{P}$ payments with social media services (e.g., in the case of Messenger payment).

This study represents our endeavor to explore these issues. Specifically, we focus on two research questions:

RQ1: Why are people unwilling to use Facebook 

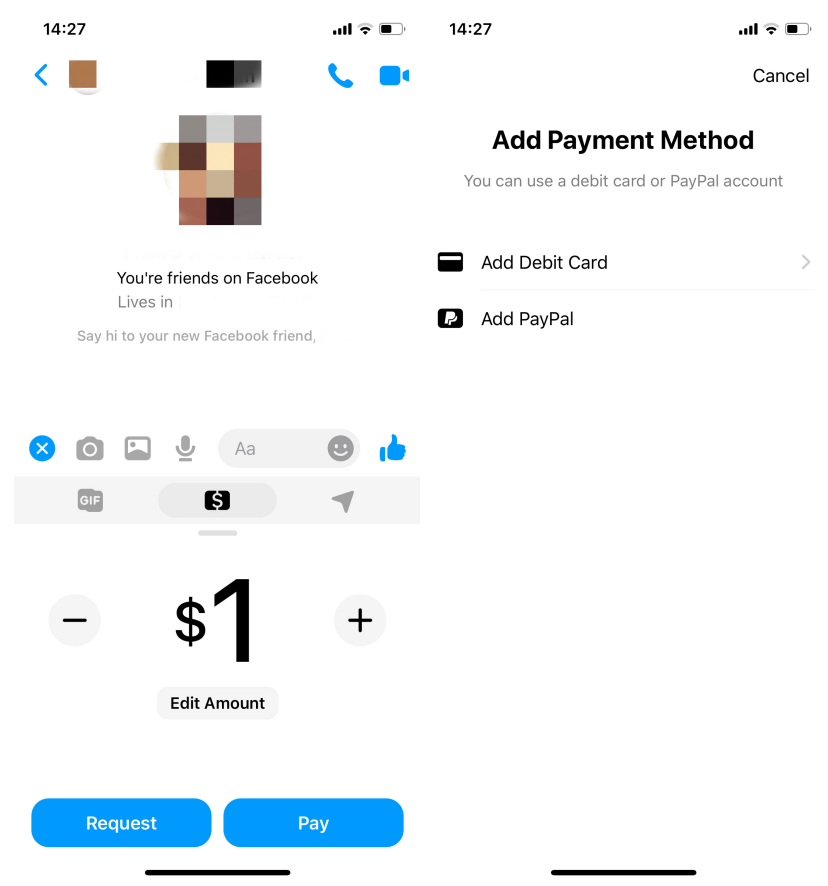

Figure 1. The payment feature in Messenger

Figure 2. Adding payment methods in Messenger

\section{Messenger payment?}

RQ2: How do people perceive the integration of $P 2 P$ payments with social media services in general?

In doing so, we qualitatively analyzed 158 social media posts and eight interviews with participants who had experience with Messenger payment. We then identified factors that hindered people from using Messenger payment and their perceptions of integrating $\mathrm{P} 2 \mathrm{P}$ payments with social media.

\section{Related Work}

\subsection{Technology Non-Use}

User and user experience of technology have always been the foci of $\mathrm{HCI}$ research. More recently, the perspective of technology non-use (e.g., why people choose not to engage in certain technology) also emerges as an important research HCI agenda [17, $18,19,20]$ since it brings together concerns regarding the appropriateness, usability, and affordability of technology.

However, there seems to be no consensus in HCI regarding how to define and contextualize technology non-use. For example, previous studies have proposed diverse ways to understand and interpret the non-use of technology. With the understanding of non-use as "the absence of a phenomenon or practice" [20],
Wyatt et al. have identified four types of non-users of the Internet: resisters, rejectors, the excluded, and the expelled [17]. Specifically, rejectors and the expelled refer to people who have used the service before but now give up voluntarily or involuntarily. Resisters and the excluded are used to describe people who have never used the service intrinsically or extrinsically [21]. Satchell and Dourish further expanded this theory to define six forms of non-use in the realm of HCI by carefully investigating the ways, aspects, and means of not using computers [19]: lagging adoption (laggards who have not started to use a technology), active resistance (hard-core who steadfastly resist to adopt a technology), disenchantment (reluctant or partial use), disenfranchisement (disenfranchised use by barriers), displacement (displaced use of a technology), and disinterest (no interest in adopting a technology). Moreover, in the field of accessible technology design, non-use involves device abandonment/discontinuance (e.g., having used but permanently giving up using) or avoidance of a device (never consider using) [22, 23].

With the rapid development of various social media platforms/services and their increasing integration with people's everyday life, a body of literature has also explored the non-use of popular social media sites such as Facebook. Collectively, these studies have shown that the decision not to use social media may arise from a variety of individual traits such as personalities [24, 25] and personal preferences [26] as well as social factors like external pressure, and data misuse [21].

In line with these studies, in this paper, we especially focus on the non-use of a specific peer-to-peer digital payment provided by Facebook (i.e., Facebook Messenger payment). We see this phenomenon as a valuable instance for $\mathrm{HCI}$ and digital economy studies. As we mentioned at the beginning of this paper, Facebook messenger payment is a unique technology that combines financial activities with social media. An empirical investigation of how and why people choose not to use it would contribute to a better understanding of technology non-use in a new research context.

\subsection{Peer-to-Peer Payment, Social Media, and HCI}

Today digital peer-to-peer (P2P) payment has become the most popular and widely accepted way to make money transactions [27]. EMarketer, a market research company, predicted that the number of people using P2P transactions would climb to 7.3 billion by 2020 [2]. More specifically, according to a new NerdWallet survey conducted in January 2020, 79\% Americans use mobile payment apps. While 
professional mobile $\mathrm{P} 2 \mathrm{P}$ payment apps such as Venmo, Zelle, and Square Cash continue to dominate this market, another growing trend is to integrate $\mathrm{P} 2 \mathrm{P}$ payments with social media services. On the one hand, popular P2P apps including Venmo and Alipay have introduced some social media features such as transaction feed [28] and messaging [29] into their products. On the other hand, established social media services have also added P2P payments to their ecosystems though very few have been successful. For instance, Snapchat allowed its users to type a dollar sign in the chatbox to trigger a money transaction between two users (i.e., Snapcash) [30]. However, this service was mainly used to pay for private content due to Snapchat's feature of disappearing after reading [31]. Snapcash only lasted for four years and was discontinued in 2018. It should be noted that one of the few successful cases is Wechat. As the leading social media app in China, it has over one billion monthly active users [32]. Wechat successfully introduced its in-app P2P payment that was built upon an important concept of the traditional Chinese culture - red envelope gifting during Chinese New Year [33, 34].

As these various forms of $\mathrm{P} 2 \mathrm{P}$ payments rise to represent our fast-paced, instant-gratification, and mobile-oriented modern life [35], they have also drawn research attention in the HCI community to understand better the role of money, experiences of mobile transactions, and financial interactions in our technology life. For example, Kaye et al. pointed out the importance of studying social, technical, and economic aspects of everyday interactions with money and emerging financial technologies and systems [13]. Chun-Wei Chiang et al. and Matsumoto et al. focused on how interface design might affect user adoption of mobile money and mobile $\mathrm{P} 2 \mathrm{P}$ payment experiences $[14,15]$. Others highlighted that factors such as the intensity of mobile phone activity and characteristics of the customer's social network could predict the future usage of mobile money [16]. And some also explored the potential privacy and security issues related to $\mathrm{P} 2 \mathrm{P}$ payments [36, 37].

Yet previous studies also reveal two main issues regarding interactions surrounding emerging financial technologies and experiences. First, many studies emphasize factors and designs that promote the use of $\mathrm{P} 2 \mathrm{P}$ payment services $[14,15,16]$. However, little is known about why people decide not to use certain forms of P2P payments (i.e., technology non-use) and why such designs and services fail. Second, limited work has been done to explore how people perceive the increasing trend of integrating P2P payments with social media services and the social consequences of such integration, except for a small body of work that exclusively focuses on Wechat [33, 34].

In this work, we attend to the unsuccessful story of Facebook Messenger payment, a seeming convenient, simple, and promising P2P payments with a potential enormous user base to address these issues by exploring reasons why people are unwilling to use Facebook Messenger payment (RQ1) and their perceptions of the integration of $\mathrm{P} 2 \mathrm{P}$ payments with social media services (RQ2).

\section{Methodology}

Data Collection Two types of data were collected in this study. We used exclusive sampling to collect social media posts and comments. First, we used keyword searches (e.g., "Messenger payment", "Facebook Messenger payment" and "social media P2P payments") on Google to collect people's self-reports regarding their views, experiences, and attitudes toward Messenger payment and the design of integrating P2P payments with social media that were posted on any online website or platform. We did not limit our search for any specific dates or years. Initially, we collected all posts and threads that Google retrieved. After filtering irrelevant posts (e.g., a description of how Messenger payment works; or emoji only), 158 valid posts and comments that explicitly expressed people's specific opinions from online forums (e.g., Reddit), news sites (e.g., CNET), and social media (e.g., Facebook) were collected.

Second, we posted a recruitment message on Facebook and Reddit to recruit interviewees who had experienced Messenger payment and who were willing to be interviewed as voluntary participants. A snowball sampling was also used. All respondents who agreed to participate were interviewed. As a result, eight semi-structured in-depth interviews were conducted via text or Face-to-Face chat based on interviewees' modality preference in November 2019. Interviews lasted from 30 to 40 minutes. Interviewees held diverse occupations (e.g., student, pastor, and engineer) and aged 22 to 41 years old. Four of them are female, and four are male. All interviewees were located in the USA. Table 1 summarizes interviewees' demographics.

Interviews started with demographic questions and focused on what and why people liked and/or disliked about Messenger payment. Example questions included "What advantages/disadvantages do you think Messenger payment has over other payment apps?", "What do you think about integrating P2P with social media such as Facebook?" and "How, if at all, did you decide not to use Messenger payment?". 
Table 1. Interviewees' Demographics

\begin{tabular}{cccc}
\hline & Gender & Age & Occupation \\
\hline I1 & F & 29 & software engineer \\
I2 & F & 24 & remote client specialist \\
I3 & M & 22 & software engineer \\
I4 & F & 23 & student \\
I5 & M & 23 & student \\
I6 & M & 22 & student \\
I7 & F & 23 & process engineer \\
I8 & M & 41 & pastor \\
\hline
\end{tabular}

Data Analysis We then used an empirical, in-depth qualitative analysis [38] of the collected data to explore people's views of using/ not using Messenger payment and attitudes toward integrating P2P payments with social media. We sought first-person, subjective, narrative accounts of their experiences in the social media posts and interviews. Our analytical procedures focused on eventually yielding concepts and themes (recurrent topics or meanings that represent a phenomenon) rather than agreement - because even if coders agreed on codes, they might interpret the meaning of those codes differently [39]. Therefore, we did not seek inter-rater reliability in our analysis but endeavored to identify recurring themes of interest, detect relationships among them, and organize them into clusters of more complex and broader themes.

Both authors reviewed the whole sample and conducted data analysis. Specifically, our analytical procedures were: 1) we closely read through the collected data to acquire a sense of the whole picture as regards how people perceive and experience Messenger payment and collectively identified thematic topics and common features in the data (e.g., purposes, advantages, disadvantages, and challenges) for further analysis; 2) we carefully examined and reviewed the thematic topics (e.g., the way people knew Messenger payment) and developed sub-themes (e.g., late into the market; established network already on other payment apps); 3) we collaborated in an iterative coding process to discuss, combine, and refine themes and features to generate a rich description synthesizing participants' perceptions and experiences of Facebook Payment and attitudes toward integrating P2P with social media.

\section{Findings}

Overall, our participants expressed their unwillingness to use Messenger payment - though they had encountered and/or experienced this service before, none was using it as of the time of the interview.
In addition, more than half $(58.2 \%)$ of the social media posts showed negative attitudes toward Messenger payment. In this section, we divide our findings into two parts. We first unpack factors that hinder people from using Messenger payment (RQ1). We then explore how such unwillingness may represent people's attitudes toward integrating $\mathrm{P} 2 \mathrm{P}$ payments with social media services (RQ2).

\subsection{Sociotechnical Barriers for Using Messenger Payment}

Our participants highlighted three main sociotechnical barriers that hindered them from using Messenger payment: the established "stickness" with other digital P2P payment methods, the existing distrust of the Facebook ecosystem, and tensions between financial transaction and personal interaction within the same platform.

\subsubsection{Established "stickiness" with Other} Competitive P2P Payment Apps When Facebook entered the already crowded P2P payment market by introducing Messenger payment in 2015, it was still considered a powerful competitor to PayPal or Venmo by mass media [40]. However, the actual users expressed strong reluctance to switch to a new platform due to the so-called app stickiness (i.e., app user engagement that leads to retention and growth; see [41]): as they had already been using other $\mathrm{P} 2 \mathrm{P}$ payment apps, they had established their routine interaction circles and expectations for $\mathrm{P} 2 \mathrm{P}$ payment services within such apps. In some sense, this form of Messenger payment non-use may result from displacement (i.e., displaced use of a similar technology) [19]: the use inertia, as one key determinant of continuance intention [42], offers comfort and reassurance to make users stay with payments apps that they have already been using for a long time.

I3 (male, 22, software engineer) believed that this psychology was prevalent among $\mathrm{P} 2 \mathrm{P}$ payment users as it partly reduced the trouble of payment apps mismatch and the peer pressure that may be brought or caused by using new apps: "I don't see myself using it unless there's a lot of people on board."

I4 (female, 23, student) also shared why it was difficult to switch to a new digital payment product, especially if the new product did not show obvious advantages over existing platforms:

"I always used Venmo. And Zelle is much more convenient now because you can send money directly to your contacts through your bank instead of adding your 
bank account info. So I prefer Zelle. In Zelle, you don't need to since it's provided by your bank."

Meanwhile, the failure to make Messenger payment visible/noticeable to its potential users made it even more impossible to persuade people to switch. For example, a comment on Quora explained,

"I think one of the main reasons why things like Facebook Messenger and Snapcash have not caught on is a lack of awareness."

In our study, only one interviewee knew Messenger payment from the news while others found out about this feature accidentally by themselves or were told by friends:

"As I was chatting with my friend and I talked about sending money, an option popped up to allow me to use messenger payment and also sometimes I misclicked on it." (I3, male, 22, software engineer)

Apparently, even if the payment function was set to appear automatically at a specific moment for users, it obviously did not draw much awareness and attention. Due to the lack of publicity, it is challenging for Messenger users to realize that the pop-up information indicated the new payment feature. I8 (male, 41, pastor) explained:

"I think I may have been talking to someone about buying an item. And I saw a little bar saying 'use it to pay'. I ignored it and continued the conversation."

In this sense, participants who had not experienced the pop-up notification in Messenger constantly complained that the feature of "payment" was "buried" in the Messenger interface. This made them see no point of taking efforts to use a new P2P payment service if few people could even know or find this feature.

\subsubsection{Distrust of the Facebook Ecosystem} Another important barrier was that people just did not trust the Facebook ecosystem. These users were much concerned about Facebook's negative reputation regarding privacy and security risks, especially after several data privacy scandals such as the case of Cambridge Analytica. As a result, participants showed active resistance [19] to the idea of combining Facebook with sensitive financial information/activities. Instead, they would actively seek alternatives, such as Zelle, a bank-supported P2P payment app. For example, a post on Cyberheist News anticipated the potential privacy and security risks of Messenger payment:

"This new Facebook payment option could allow several kinds of scams....Also, when a friend messages you and their account has been hacked, there is a criminal trying to scam you impersonating your friend."

According to this post, Messenger payment may inherently subject to the vulnerabilities of the instant messaging app itself. Therefore, a reasonable alternative was to use other specialized professional payment apps.

Our interviewees also echoed this concern: I5 (male, 23, student) equaled Messenger payments section to the entire Facebook:

"I wouldn't want to put my information, my credit or debit card information into something that is basically FB just out of security reasons."

I5 was afraid of using Messenger payment simply because this service was provided by Facebook. For him, his distrust of Facebook, in general, was valid enough to motivate him not to use a digital payment affiliated with it.

More importantly, other popular digital P2P platforms such as Zelle partners with leading banks and credit unions, improve their credibility to manage and protect financial activities. In contrast, the lack of a trustworthy financial entity as endorsement and support behind Facebook leads to increasing privacy and security concerns, as I8 said, "Who is the bank for Facebook, I don't know."

In fact, Messenger payment did provide extra security procedures such as PIN or using facial recognition or fingerprint to confirm transactions. However, regardless of these effective security features, Facebook's negative publicity in the media has made such security settings less convincing, deepening people's distrust of Facebook's products. Many potential users seemed to be already cautious about the Facebook ecosystem, making them reluctant to try any financial services provided within this ecosystem.

4.1.3. Tensions between Financial Transaction and Personal Interaction Another interesting finding was that people considered combining financial transactions with Messenger, an instant messaging app for personal interaction, quite awkward. As a result, these users were reluctant to use or were only willing to partly use, Messenger payment as disenchantment [19]. A post on Reddit explained that using $\mathrm{P} 2 \mathrm{P}$ payment through an app that was for chatting and connecting with friends was embarrassing, especially when it came to borrowing money: "All it's going to lead to is people I haven't seen since high school asking me to lend them money."

Other comments on Facebook also sarcastically expressed the underlying concerns regarding conveniently integrating financial interaction in social interaction: " $U$ know my birthday is coming up..." "Doug, let me borrow 500 dollars." For some users, this awkward combination often leads to negative experiences, as a Facebook comment showed: 
"This is awful. FACEBOOK you are opening a can of worms that will cause a lot of friends to part ways."

Therefore, some participants expressed the hope to avoid intertwining social and financial lives. For example, I4 (female, 23, student) said, "I just prefer to talk with friends on it. With payment apps, they're only for payment."

For many people, Facebook is always viewed as a platform to establish or maintain close social relationships (e.g., friends, classmates, alumni, or family members). Messenger makes such social interactions convenient and direct by offering private and immediate one-to-one interpersonal communication. Conducting financial activities such as $\mathrm{P} 2 \mathrm{P}$ payment in a sociability and communication-centric platform seems inappropriate for them - would an instant messaging app be professional and safe enough to deal with financial activities? How would conducting financial activities through an instant messaging app lead to potential tensions and undesired social consequences (e.g., how to decline a friend's message to borrow money through Messenger payment)? Therefore, an effective and simplest solution is not to use Messenger payment to avoid potential embarrassment or destruction of friendships.

\subsection{Diverse Perceptions of Integrating P2P Payments with Social Media}

Obviously, Facebook was not the first to integrate $\mathrm{P} 2 \mathrm{P}$ payments with social media services in the world. While Messenger payment's path to success seems not to be smooth in the USA, Wechat's P2P payment service has dominated China's $\mathrm{P} 2 \mathrm{P}$ payment market. As integrating $\mathrm{P} 2 \mathrm{P}$ payments and social media becomes an emerging trend for innovating financial technologies/services, it is important to understand people's diverse perceptions of such an integration.

In our study, some participants did hold a more positive attitude towards this trend. A few social media posts described it as "a brilliant move" or "a great advance" (Facebook comments). I8 (male, 41, pastor) was also impressed by its novelty: "I think it's a creative idea. And I think many people would probably use it eventually."

Some others highly praised the convenience of such an integration for everyday life. For example, I6 (male, 22, student) mentioned:

"It's totally ok to combine social media with payment, right? And maybe this could be even more convenient for us, because lots of our friends or network are already on board."

For him, the biggest benefit was his existing social network on the platform (e.g., "lots of our friends or network are already on board"). Compared to other digital $\mathrm{P} 2 \mathrm{P}$ payment services where users have to manually add recipients, integrating $\mathrm{P} 2 \mathrm{P}$ payments with social media takes advantage of a user's existing social network, which would significantly save one's time and energy to establish the financial network.

A Reddit post showed similar thoughts:

"For someone like me who uses Messenger a whole lot for different reasons, it's nice to see all these features."

For these users, a comprehensive ecosystem that incorporates diverse functions would provide them with greater convenience - e.g., no need to download standalone apps one by one.

However, some others felt quite overwhelmed by such an all-in-one integration. From the perspective of design, a post on The Verge expressed concerns regarding incorporating excess functions in one platform, which may lead to information overload: "I rather see separate apps for these kind of things, mobile apps shouldn't be so bloated".

Others also considered this integration "too ambitious" and as a privacy invasion: "They want to get every piece of info about us!" (Facebook comment)

Especially, a post on Reddit well summarized why people should avoid this trend of all-in-one design:

"They want to be a part of every single moment of a person's life. [...] They want all your info, everything about your day both online and offline, they want to be your bank, they want... THEY WANT EVERYTHING."

In summary, integrating $\mathrm{P} 2 \mathrm{P}$ payments with social media appears to be a double-edged sword: while some may find it more convenient by affording all essential everyday social activities (e.g., networking, communication, and paying bills) through a single platform, others express concerns about information overload, higher privacy and security risks, and confusions/tensions between different social functions.

\section{Discussion}

To answer our research questions, our findings have shown that: 1) the reasons why people are unwilling to use Messenger payment lie in the established "stickiness" with other P2P payment apps, Facebook itself, and the tensions between financial transactions and personal interaction (RQ1); 2) participants in our sample showed diverse perceptions of integrating P2P payments with social media including praise for its convenience to afford essential financial and social activities through a single platform, concerns about information overload and privacy/security risks, and 
confusions regarding different social functions (RQ2).

New Perspectives on Technology Non-Use. Our study confirms with previous research on the social and cultural reasons for technology non-use [21, 17, 43, 44]. Participants have shown proactive attitudes to refuse to adopt Messenger payment. These proactive attitudes also help shape cultural interpretations of technology as well as eager adopters do [19]. As active resisters, they expressed critical concerns and alternatives toward Messenger payment. Specifically, our results suggest that privacy [25], data misuse [21], and preference for other ways [26] lead to non-use as prior studies concluded.

However, the non-use of Messenger payment is also uniquely intertwined with Facebook as a social media platform. This indicates one's struggles between financial life and social life, which leads to potential non-use. As we have shown, incorporating financial functions with social functions (e.g., social networking and instant messaging) led to tensions between financial transactions and personal interactions. This unique combination triggered feelings of awkwardness and embarrassment, which motivated them not to use such a service. Due to the fact that all-in-one design has just become a popular technology trend in recent years, how the integration of different technologies and social functions within one platform/application further extends and complicates technology non-use would be an important question that requires future research.

Consistent with previous studies [14, 15, 16], our findings also show that design plays a crucial role in encouraging or discouraging the use of $\mathrm{P} 2 \mathrm{P}$ payment services. For example, the design decision to hide the payment feature in the Messenger interface led to poor publicity and low awareness of this service, which resulted in its failure.

Yet, our findings reveal that other sociocultural factors beyond design would affect P2P payment users' decision-making. This provides new insights on technology non-use in the context of financial technologies. For example, prior studies show that non-users were dissatisfied with the specific technology or service itself. In contrast, in our study, people decided not to use Messenger payment due to their existing privacy and security concerns about Facebook as an ecosystem rather than about Messenger payment itself. Therefore, the social image of the brand or the tech ecosystem itself matters. In the case of Messenger payment, it suffered from a damaged public image of Facebook regarding data privacy breaches and security risks. According to $\mathrm{Fu}$ et al., user-perceived security and privacy concerns and their stereotypes often led to the rejection of the chat function in the online payment app Alipay [29]. Similarly, in our study, Facebook's damaged public image invited distrust and security/privacy concerns. It also reinforced the stereotype that using social media to make payment was insecure and troublesome. In this sense, sociocultural factors such as the perceived privacy and security considerations of a technology's social image lead to potential users' low faith in using its service, even though the technology giant implemented highly advanced security features. Sociocultural facotrs, therefore, in addition to design patterns, should also be taken into account when developing sustainable digital payment system.

Lessons for All-in-One Design. Our sample is relatively small. Yet our participants still provided certain insights that may inform plausible future directions for better approaching and promoting all-in-one design - an increasingly popular trend in technology design that integrates multiple diverse functions within one application.

First, our participants pointed out that an appropriate entry point to guide users to accept and adopt all-in-one design matters. In this case of Messenger payment, incorporating this new P2P payment service with social media seemed to be abrupt. It lacked an appropriate presentation of how this design concept could be closely related to users' everyday lives (e.g., how could Messenger payment be closely tied to users' daily social activities and interactions with friends?). In contrast, the success of Wechat P2P payment may partially lie in its appropriate first introduction - P2P payment as red envelopes (monetary gifting) for the Chinese New Year, which is not only seamlessly interwoven with daily lives but also deeply embedded in the traditional Chinese culture that most users are familiar with [33, 34]. In this sense, framing financial transactions as "red pocket" through WeChat fits Chinese users' cultural expectations for financial activities. This helps them embrace the integration of money transactions with social media (as a form of all-in-one design) more comfortably. In contrast, directly gifting money for social events may be generally considered inappropriate in Western cultures. As a result, the "red pocket" model may not work in platforms in the US or other Western cultures. This seems to highlight the importance for designers and developers who engage with all-in-one design to take local cultures into account. For example, marketing strategies for introducing any all-in-one design products should be designed and implemented according to specific local cultures' values.

Second, all-in-one design appears to be both convenient and comprehensive (e.g., using one platform to access all features/functions needed) for some of 
our participants. However, it may trigger unwanted confusion and tensions for some others due to the blurred boundaries between different functionalities (e.g., switching between different features, conducting financial activities while maintaining everyday social networking). Such blurred boundaries often affect users' strategies when coping with various task-oriented functions and thus increase usage workload. This becomes a major challenge for people to accept and adopt all-in-one design.

In our study, Facebook Messenger as an instant messaging tool, facilitates frequent and close interpersonal communication. This sometimes makes social interactions too close and too crowded for users [45]. In addition, it is not an ideal environment for financial activities as people tend to be more cautious and prudent when conducting such activities. In this sense, Messenger payment endeavors to host, support, and maintain two different contexts of interactive activities within one platform.

On the one hand, this integration does allow Facebook users to initiate payment behaviors more directly and easily. On the other hand, it also greatly increases the uncertainties, tensions, and confusion. For example, which social norms or regulations should users follow to deal with the intertwined financial/social activities?

As our study has shown, when participants were asked to lend money to those with whom they had weak ties on Messenger, they not only had to make judgments about trust based on one's developed social capital and the platform itself [46] but also were forced to face their own roles in an awkward social relationship (e.g., lending money to build a stronger relationship or not lending to risk a potentially strong relationship). Therefore, it is important for $\mathrm{HCI}$ designers to carefully tease out the blurring boundaries of different functionalities, the specific social context of individual functionality, as well as users' diverse needs in order to make future all-in-one designs more acceptable to users.

Limitations and Future Work. This study has three limitations. First, our sample size is relatively small. To provide comprehensive understandings of how people perceive the integration of social media and $\mathrm{P} 2 \mathrm{P}$ payment, we plan to recruit a larger sample with more diverse backgrounds in future work. Second, the sample is also engineering-centric. People in different domains/occupations may perceive and use digital P2P payments differently. Our future work will address the limitation and focus on recruiting participants in various domains/occupations to verify this study's findings. Third, our sample focused on people's opinions on digital payment and all-in-one design in the United States. For future work, we plan to conduct a cross-cultural study of all-in-one design involving users with different cultural backgrounds (e.g., North American and East Asian) to further explore the sociocultural factors that affect such design.

\section{Conclusion}

In this paper, we have explored why people choose not to use Facebook Messenger payment even though Facebook has achieved a huge success to win a large user base. Our findings have shown that established "stickiness" with other competitive P2P payment apps, perceived distrust of Facebook ecosystem and reputation, and tension emerging in the intertwined social and financial interaction make people unwilling to try or continue using the service. We have also highlighted diverse perceptions of integrating P2P payments with social media. The perceptions will help inform future research on the usability of all-in-one design. We believe that these findings extend our existing understanding of technology non-use through the lens of Facebook payment. We also hope that this study opens up opportunities for HCI researchers and designers to innovate emerging financial technologies and develop all-in-one technological platforms in the future.

\section{References}

[1] D. Fuscaldo, "Here's an area of digital payments that's booming: P2p payments," Forbes. August, 2019.

[2] A. He, "Strong growth from venmo and zelle drives p2p transaction volume," eMarketer, 2019. November.

[3] L. Grilli, "P2p - a comprehensive look at person-to-person payments," $2017 . \quad$ http: //www.thepaymentsreview.com/ $\mathrm{a}-100 \mathrm{k}-\mathrm{at}-\mathrm{p} 2 \mathrm{p}$-payments.

[4] S. Chung, "How entitled millenials send payments," 2017. https://abovethelaw.com/2017/07/ how-entitled-millenials-send-payments / ? rf $=1$.

[5] H. Jacobs, "One photo shows that china is already in a cashless future,” Business Insider, 2018.

[6] Tencent, "Tencent announces 2019 fourth quarter and annual results," Tencent Global, 2020.

[7] P. Allan, "You can now use facebook messenger without a facebook account," Lifehacker, 2015. June.

[8] I. Hundrev, "How to set up facebook payments in messenger," Learning Hub. February, 2019.

[9] E. Protalinski, "Facebook passes 1.44 b monthly active users and 1.25 b mobile users; $65 \%$ are now daily users," Venture Beat, 2015.

[10] E. Protalinski, "Facebook messenger passes $600 \mathrm{~m}$ monthly active users as growth keeps up with messaging leader whatsapp," Venture Beat, 2015. 
[11] J. Clement, "U.s. facebook messenger payments payment app usage frequency 2017," Statista. November, 2017.

[12] I. Lunden, "Facebook is discontinuing p2p payments in the uk and france on june 15," TechCrunch. April, 2019.

[13] J. Kaye, J. Vertesi, J. Ferreira, B. Brown, and M. Perry, "\#chimoney: Financial interactions, digital cash, capital exchange and mobile money," in CHI'14 Extended Abstracts on Human Factors in Computing Systems, pp. 111-114, ACM, 2014.

[14] C.-W. Chiang, C. Anderson, C. Flores-Saviaga, E. Arenas Jr, F. Colin, M. Romero, C. Rivera-Loaiza, N. E. Chavez, and S. Savage, "Understanding interface design and mobile money perceptions in latin america," in Proceedings of the 8th Latin American Conference on Human-Computer Interaction, p. 5, ACM, 2017.

[15] M. Matsumoto and L. Terrenghi, "Learning from emerging and mature markets to design mobile $\mathrm{p} 2 \mathrm{p}$ payment experiences," in International Conference on HCI in Business, Government, and Organizations, pp. 238-247, Springer, 2016.

[16] S. Centellegher, G. Miritello, D. Villatoro, D. Parameshwar, B. Lepri, and N. Oliver, "Mobile money: Understanding and predicting its adoption and use in a developing economy," Proceedings of the ACM on Interactive, Mobile, Wearable and Ubiquitous Technologies, vol. 2, no. 4, p. 157, 2018.

[17] S. M. Wyatt, "Non-users also matter: The construction of users and non-users of the internet," Now users matter: The co-construction of users and technology, pp. 67-79, 2003.

[18] E. P. Baumer, M. G. Ames, J. R. Brubaker, J. Burrell, and P. Dourish, "Refusing, limiting, departing: why we should study technology non-use," in CHI'14 Extended Abstracts on Human Factors in Computing Systems, pp. 65-68, 2014.

[19] C. Satchell and P. Dourish, "Beyond the user: use and non-use in hci," in Proceedings of the 21st Annual Conference of the Australian Computer-Human Interaction Special Interest Group: Design: Open 24/7, pp. 9-16, 2009.

[20] E. P. Baumer, J. Burrell, M. G. Ames, J. R. Brubaker, and P. Dourish, "On the importance and implications of studying technology non-use," interactions, vol. 22, no. 2 , pp. 52-56, 2015.

[21] E. P. Baumer, P. Adams, V. D. Khovanskaya, T. C. Liao, M. E. Smith, V. Schwanda Sosik, and K. Williams, "Limiting, leaving, and (re) lapsing: an exploration of facebook non-use practices and experiences," in Proceedings of the SIGCHI conference on human factors in computing systems, pp. 3257-3266, 2013.

[22] M. J. Scherer, Living in the state of stuck: How assistive technology impacts the lives of people with disabilities. Brookline Books, 2005.

[23] R. Grott, "On technology abandonment or discontinuance," in Assistive Technology Service Delivery, pp. 211-216, Elsevier, 2019.

[24] T. Ryan and S. Xenos, "Who uses facebook? an investigation into the relationship between the big five, shyness, narcissism, loneliness, and facebook usage," Computers in human behavior, vol. 27 , no. 5 , pp. 1658-1664, 2011.
[25] S. Stieger, C. Burger, M. Bohn, and M. Voracek, "Who commits virtual identity suicide? differences in privacy concerns, internet addiction, and personality between facebook users and quitters," Cyberpsychology, Behavior, and Social Networking, vol. 16, no. 9, pp. 629-634, 2013.

[26] R. K. Baker and K. M. White, "In their own words: Why teenagers don't use social networking sites," Cyberpsychology, Behavior, and Social Networking, vol. 14, no. 6, pp. 395-398, 2011.

[27] L. Huang and J. Savary, "When payments go social: The use of person-to-person payment methods attenuates the endowment effect," Available at SSRN 3469706, 2019.

[28] A. Acker and D. Murthy, "Venmo: Understanding mobile payments as social media," in Proceedings of the 9th International Conference on Social Media and Society, pp. 5-12, ACM, 2018.

[29] J. Fu, Y. Zhou, S. Wu, and X. Wang, "Let's chat in alipay: Understanding social function usage in task-oriented apps," in Extended Abstracts of the 2019 CHI Conference on Human Factors in Computing Systems, p. LBW0276, ACM, 2019.

[30] G. David, "4. all what we send is selfie: Images in the age of immediate reproduction," Mobile and Digital Communication, pp. 79-100, 2015.

[31] J. Constine, "Snapchat will shut down snapcash, forfeiting to venmo," TechCrunch. July, 2018.

[32] M. Iqbal, "Wechat revenue and usage statistics (2019)," Business of Apps, vol. 27, 2019.

[33] W. Xu, "The study of wechat payment users willingness factor," Journal of Service Science and Management, vol. 10, no. 3, pp. 251-259, 2017

[34] Z. Wu and X. Ma, "Money as a social currency to manage group dynamics: Red packet gifting in chinese online communities," in Proceedings of the 2017 CHI Conference Extended Abstracts on Human Factors in Computing Systems, pp. 2240-2247, ACM, 2017.

[35] C. Newlove, "The rise of peer-to-peer (p2p) payments on mobile - what makes it such a crucial feature?," Medium, 2018. August.

[36] M. M. Jozani, E. Ayaburi, M. Ko, and K.-K. R. Choo, "A conceptual model to understand privacy concerns in a social p2p payment app," 2018.

[37] S. Khandekar, J. Liang, A. Razaque, F. Amsaad, and M. Abdulgader, "Security research of a social payment app and suggested improvement," Communications on Applied Electronics (CAE), 2016.

[38] A. L. Strauss, Qualitative analysis for social scientists. Cambridge university press, 1987.

[39] N. McDonald, S. Schoenebeck, and A. Forte, "Reliability and inter-rater reliability in qualitative research: Norms and guidelines for cscw and hci practice," Proceedings of the ACM on Human-Computer Interaction, vol. 3, no. CSCW, pp. 1-23, 2019.

[40] J. Constine, "Facebook introduces free friend-to-friend payments through messages," TechCrunch. March, vol. 17,2015 .

[41] C.-L. Hsu and J. C.-C. Lin, "Effect of perceived value and social influences on mobile app stickiness and in-app purchase intention," Technological Forecasting and Social Change, vol. 108, pp. 42-53, 2016. 
[42] W.-T. Wang, W.-M. Ou, and W.-Y. Chen, "The impact of inertia and user satisfaction on the continuance intentions to use mobile communication applications: A mobile service quality perspective," International Journal of Information Management, vol. 44, pp. 178-193, 2019.

[43] R. Kline, "Resisting consumer technology in rural america: The telephone and electrification," How users matter: The co-construction of users and technology, pp. 51-66, 2003.

[44] A. S. Laegran, "Escape vehicles? the internet and the automobile in a local-global intersection," How users matter. The co-construction of users and technology. Cambridge (Mass.): MIT Press. THE RESEARCHING AND TEACHING COMMUNICATION SERIES, vol. 200, pp. 81-100, 2003.

[45] I.-g. Shin, J.-m. Seok, and Y.-k. Lim, "Too close and crowded: Understanding stress on mobile instant messengers based on proxemics," in Proceedings of the 2018 CHI Conference on Human Factors in Computing Systems, pp. 1-12, 2018.

[46] M. Nouwens, C. F. Griggio, and W. E. Mackay, “” whatsapp is for family; messenger is for friends" communication places in app ecosystems," in Proceedings of the 2017 CHI conference on human factors in computing systems, pp. 727-735, 2017. 